\title{
MATEAYUDA: Una herramienta tecnológica para reforzar aprendizajes matemáticos
}

Jairo José Flores Morales ${ }^{1}$

1 UNAN-Managua,FAREM-Chontales.Correo Electrónico: jairofmdjmix@yahoo.com

\section{RESUMEN}

La tecnología de las comunicaciones pone a nuestro alcance diversos recursos que pueden ser utilizados con distintos fines, entre ellos los educativos. Las herramientas virtuales que hoy en día ofrece internet, permiten un entorno virtual de aprendizaje caracterizado por la participación e interacción colaborativa entre sus miembros, conformando lo que hoy se conoce como la "Web 2.0".

Este trabajo presenta una experiencia innovadora con estudiantes universitarios, al usar una página en Facebook como una potente herramienta para reforzar aprendizajes en la asignatura de Matemática General, demostrando su utilidad a través de los resultados obtenidos. Además, se brindan percepciones estudiantiles sobre el uso de las redes sociales en el ámbito educativo, la ayuda significativa ofrecida con la página creada, las interacciones entre docente-estudiante y el alcance logrado durante el curso.

Palabras clave: Innovación, red social, Facebook, tecnología educativa, didáctica. 


\title{
MATEAYUDA: A technological tool for reinforcing math learning
}

Jairo José Flores Morales ${ }^{1}$

1 UNAN-Managua,FAREM-Chontales.E-mail: jairofmdjmix@yahoo.com

\begin{abstract}
Communications technology provides us various resources that can be used for various purposes, including education. The virtual tools that today provides Internet, allow virtual learning environment characterized by collaborative participation and interaction among members, forming what is known today as the "Web 2.0".

This paper presents an innovative experience with college students, using a Facebook page as a powerful tool to enhance learning in the subject of General Mathematics, proving its worth through the results. In addition, provide student perceptions on the use of social networking in education, offered significant support to the page created, interactions between teacher-student and scope achieved during the course.
\end{abstract}

Keywords: Innovation, social network, Facebook, educational technology, didactic. 


\section{INTRODUCCIÓN}

Para nadie es un secreto el impacto que internet ha teniendo en nuestra sociedad, significando una herramienta con innumerables ventajas en cuanto al suministro de plataformas de información en diversos campos de la vida.

Las redes sociales han crecido exponencialmente, por ejemplo Facebook hasta julio del 2013 contabilizaba 1,150 mil millones de usuarios activos en el mundo, cifra que aumenta conforme pasan los meses. Esta realidad abre la puerta para utilizar dichas redes sociales en el campo educativo, aspecto que muy pocos se han adentrado a estudiar.

Ahora bien, para evaluar si la red social Facebook cuenta con características que permitan su uso como plataforma educativa y para actividades académicas colaborativas, cabe indagar ¿Qué ventajas, potencialidades y limitaciones presenta Facebook para un posible uso educativo de carácter colaborativo? Es ahí donde surge la idea de crear un sitio web para apoyar significativamente al estudiantado universitario en sus aprendizajes matemáticos, dejando a un lado la monotonía de aprender bajo las mismas condiciones que lo hacían en el siglo pasado y que lamentablemente aún se sigue haciendo, pese a las múltiples tecnologías que tenemos en la actualidad.

Muy acertadamente Freire (2005:78-79) expone la necesidad de cambiar tanto la relación alumnodocente como la creatividad en la enseñanza al decir:

"Educadores y educandos se archivan en la medida en que, en esta visión distorsionada de la educación, no existe creatividad alguna, no existe transformación ni saber. Sólo existe saber en la invención, en la reinvención, en la búsqueda inquieta, impaciente, permanente que los hombres realizan en el mundo ... La educación debe comenzar por la superación de la contradicción educador-educando."
Ciertamente, el educador que no inventa, que no se atreve, que no utiliza las herramientas que internet ofrece, jamás favorecerá la educación pertinente y holística que los jóvenes universitarios necesitan para enfrentar los retos que la sociedad moderna les tiene preparado.

Las redes sociales han cambiado la forma en que nos comunicamos y compartimos información. Millones de personas usan cada día distintos sitios web de redes sociales, de manera que éstas ya forman parte de nuestra vida cotidiana. No hay día en que no se verifique en los medios de comunicación un cambio en nuestra manera de relacionarnos, propiciado por el auge de las redes sociales (Santamaría, 2009 ; Orhihuela, 2008), y por lo tanto, las Matemáticas no son una excepción.

En el Informe Horizon Edición Iberoamericana del año 2010 , citado por García (2010:4), se señala:

"La tecnología no sólo es un medio para capacitar a los estudiantes, sino que se convierte en un método de comunicación y de relación, así como una parte ubicua y transparente de su vida" ..., nuestra forma de pensar acerca de los entornos de aprendizaje está cambiando. Tradicionalmente y hasta hace poco tiempo, los entornos de aprendizaje se asociaban a espacios físicos. Hoy, sin embargo, los "espacios" donde aprenden los estudiantes son cada vez más comunitarios e interdisciplinarios yestán apoyadosportecnologías asociadas a la comunicación y a la colaboración virtual. Los espacios se transforman para hibridar lo presencial con lo virtual, difuminándose las fronteras entre ambos mundos, que son vividos por los estudiantes como uno solo"

Lo antes escrito, hace suponer la necesidad de actualizar nuestra práctica docente e involucrar a dichos recursos como parte de nuestro que hacer magisterial. 
Estudios previos demuestran la importancia de las redes sociales, entre ellas Facebook, como herramientas de enseñanza. Anguita et al. (2006) citados por Barajas y Álvarez (2013), mencionan positivamente el estudio de casos realizado con el curso 2005-2006 en la especialidad de Educación Social en la Universidad de Valladolid, grupo con el que se constituyó una comunidad de práctica usando redes sociales. Por su parte García (2008) citado siempre por Barajas y Álvarez (2013), exponen que la experiencia al desarrollar un proceso de aprendizaje colaborativo a través del Facebook, permite en gran medida, el aprovechamiento de las herramientas que brinda la Web 2.0, con alumnos de segundo año de la carrera de Periodismo en la Universidad Andrés Bello de Chile.

También Robles (2009), Domínguez (2009), Chavarro (2010), Pisticelli, Adaime y Binde (2010), citados por Barajas y Álvarez (2013), describen respectivamente el uso de la red social en el aprendizaje de inglés, la experiencia de su uso como modelo para realizar un club de revistas en la enseñanza de la medicina, experiencias con Facebook en cursos de Introducción a la Economía, dando pautas sobre cómo se puede utilizar la red Facebook en la educación, en el marco de la cátedra de introducción a la informática, telemática y al procesamiento de datos.

A pesar de estos resultados expuestos anteriormente, en nuestro país aún no se evidencia un estudio detallado sobre la utilidad de la red social en educación, razón por la cual se ha considerado, que este trabajo permitirá ampliar la visión que tenemos sobre Facebook. Recordemos que en los países en desarrollo la educación debe ser una prioridad, pues esta transforma la sociedad, pero a su vez, la tecnología tiene el poder de transformar la educación, por lo que acertadamente lo expresado por Kirchman (2010), citado por Gómez y López (2010), expone el futuro de la educación:
"Las nuevas tecnologías de la información y la comunicación evolucionaron en los últimos años de manera exponencial. La capacidad de interconexión a través de la red y programas de fácil manejo son parte de ese crecimiento. En ese sentido, las redes sociales, con su capacidad innata de crear comunidad, se perfilan como una alternativa interesante para incluir en los procesos educativos".

Más aún, Zamora (2013:7) advierte que las universidades nicaragüenses deben centrar su interés en los recursos TIC's y en las innovaciones didácticas al decir:

"Nos enfrentamos a realidades donde el alumno trae consigo una diversidad de paradigmas, escucha muchas noticias, está insertado en la generación de la internet o la llamada e-generation, donde las cuestiones fluyen a toda velocidad y mientras la universidad se siga comportando como la escuela aburrida, encerrada en modelos tradicionales de enseñanza, actitudes desfasadas del profesorado, escasa relación sociedaduniversidad, universidad-empresa, poco empleo de las TIC's como herramienta casi imprescindible en la gestión del conocimiento, de la información y del desarrollo no será posible entrarle a la discusión de la formación para la ciudadanía global. Por tanto se debe asumir que estamos en un mundo donde para desarrollarnos, sin quedarnos atrás, debemos emprender estratégicamente la gestión del conocimiento, y con ello la incorporación de las tecnologías modernas de la información".

Por tanto, se hace evidente la necesidad de realizar cambios en nuestras percepciones educativas con relación al mundo de hoy, y por ende, aprovechar la oportunidad que ofrecen los diferentes medios y herramientas educativas con que cuenta la web 2.0. 
HERRAMIENTAS EN LA EDUCACIÓN DEL SIGLO XXI.

El papel del docente hoy en día debe estar enmarcado en promover un rol eminentemente auxiliar, siendo catalizador y armonizador dentro del proceso educativo en los salones de clases, facilitando recursos innovadores y suprimiendo obstáculos que puedan presentarse en el proceso formativo (Palacios, 1997). Por su parte Tünnermann (1993:233) pone de manifiesto los cambios que se deben hacer en la educación superior al expresar:

"La educación superior contemporánea, al asumir la realidad del vertiginoso desarrollo de la ciencia y la tecnología, deberá enfatizar sobre la formación básica y general, así como también en los procesos de aprendizaje... Esto significa que la educación que se brinde deberá estimular la creatividad e imaginación, de modo que sepa encontrar, por si mismos, la respuesta a los retos que le plantearán un mundo y una ciencia en proceso constante de mutación."

Por ende, los recursos tecnológicos y diversas herramientas que actualmente posee nuestra sociedad, favorecen en gran medida que la una educación esté en constante cambio, López (2007:17) expone una necesidad cada más creciente dentro del sistema educativo universitario al decir:

"El ámbito universitario, como último escalón educativo de carácter formal, no puede quedar al margen de una realidad...., por lo que se impone cuestionar el actual rol del profesor en la enseñanza superior, así como repensar de forma seria la docencia universitaria. Hablamos de que el docente, de forma colaborativa, realice un giro significativo desde diferentes puntos de vista (pedagógico, epistemológico y psicosocial), en el que se haga indispensable la búsqueda de nuevas estrategias docentes,... y que deberían ser tenidos en cuenta en cualquier iniciativa pedagógica innovadora."

Notoriamente la educación de este siglo, no puede seguir enfrascándose en forma cíclica en un modelo centrado en los contenidos y en la tradición es fundamental comenzar a entender que la verdadera educación debe estar basada en los intereses de los estudiantes, su motivación, la utilidad que posee el nuevo aprendizaje, la riqueza del contexto, la globalización, la tecnología, y no menos importante la holística integradora que debe existir en nuestro marco educacional universitario, cuestionamientos que hoy en día deben marcar una reestructuración de lo que se hace en la educación superior.

Dicho de otra forma, tenemos que enajenar nuestra forma de enseñanza, incluyendo lo que está palpablemente en nuestras manos, como lo es la educación tecnológica y las herramientas que dichas tecnologías nos ofrecen. Por lo que el docente debe percibir con mejor perspectiva los avances modernos en esa materia. Solana (2008:80) lo expresa de la siguiente manera: "La educación tecnológica debe responder al avance del conocimiento y a sus aplicaciones en el mundo actual. Para ello, debe superar ese enfoque reduccionista con el que a veces se le califica; ubicarse en su dimensión social real, donde sus objetivos, su quehacer y su aportación al progreso, se traduzcan en valores no técnicos. Por esto, el para qué de la educación tecnológica debe ser redefinido a partir de dos paradigmas: innovación y participación".

\section{USO DE FACEBOOK EN EDUCACIÓN}

Facebook por ser una de las redes sociales en internet que más ha crecido en los últimos tiempos en lo que respecta a cantidad de usuarios, ofrece diversas posibilidades de uso en educación, permitiendo pasar del tipo de educación tradicional a sistemas revolucionarios como la "educación personalizada" 
propuestas por Salman Khan (una revelación actual en el ámbito educativo). Khan advierte que la educación está pasando de un modelo pasivo al modelo activo, fomentando modelos de clases al revés, en donde el alumno estudia en su casa y hace las tareas en el centro educativo, aprendiendo de esta forma a su propio ritmo (TED,2011).

Este pensamiento cala profundamente en los ideales novedosos en materia de educación, generando la posibilidad para que el mismo estudiante utilice los medios que gratuitamente ofrece internet, para ver publicaciones matemáticas en diversos tópicos, repetir una y otra vez la lección transmitida en videos, acto que pocas veces se realiza en las sesiones de clases, por supuesto, que este tipo de "educación invertida" aún necesita propagandización en nuestro país.

Facebook ofrece posibilidades de intercambio de información a partir de redes sociales de personas, en una interface sencilla para el usuario, un amplio acceso a la multimedia ( se pueden hacer referencias y links a videos, música y direcciones web), la posibilidad de encuentro con otros estudiantes o maestros para intercambiar ideas e información, mensajes en muros y foros de debate. Es importante ver esta red social como una herramienta con múltiples usos, entre ellos la educación, para no quedarse en la fascinación de su utilización más difundida (con fines de ocio, distracción y conexión por esparcimiento).

El mundo educativo no puede permanecer ajeno ante fenómenos sociales como este que está cambiando la forma de comunicación entre las personas. El sistema educativo trabaja fundamentalmente con información, carecería de sentido utilizar sistemas de transmisión y publicación de la misma basada en aquellos que se utilizaban a principios y mediados del siglo $\mathrm{XX}$, sin incorporar aquello que la sociedad ya está usando como parte de su vida cotidiana.

\section{MATEAYUDA: UNA PROPUESTA INNOVADORA UNIVERSITARIA}

Toda innovación educativa surge a través de un proceso gradual, buscando soluciones a diversas dificultades en el proceso de enseñanza aprendizaje. Según la definición dada por Imbernón (1996), citado por Planas (2011:60):

"la innovación educativa es la actitud y el proceso de indagación de nuevas ideas, propuestas y aportaciones efectuadas de manera colectiva, para la solución de situaciones problemáticas de la práctica, lo que comportará un cambio en los contextos y en la práctica institucional de la educación".

Por tal razón, al innovar se busca transformar el aula con nuevas metodologías, contenidos, colaboración entre docentes, teniendo en cuenta que innovar en los procesos educacionales ofrece cambios no en forma inmediata, pero sí en forma paulatina (Planas, 2011). Al realizar novedosas maneras de enseñar contenidos matemáticos y lograr aprendizajes relevantes en los estudiantes, se está logrando romper los paradigmas negativos y perdurables referentes al modelo tradicional de enseñanza matemática.

Siguiendo la misma senda, Font y Godino (2011) afirman que siempre será oportuno que el docente reflexione sobre su práctica a partir de su conocimiento del contexto educativo, basados en la didáctica de las matemáticas como medio para realizar innovaciones, que serán relevantes para la institución educativa en la que labora, y por supuesto, para el propio docente como méritos en su carrera profesional.

Es por tal razón, que la página educativa MATE AYUDA propone una metodología centrada en la interacción social que se establece desde Facebook, entre el docente y el mismo estudiante; sin obviar, el impacto que poseen los videos, siendo éstos una 
forma de reinventar la educación de nuestros tiempos.

En consecuencia, mejorar la praxis magisterial desde una perspectiva holística, involucradora y llena de conciencia social, es la misión de cualquier investigador del campo de la didáctica matemática, esa directriz es la que acompaña a todo este trabajo investigativo, a lo largo de sus etapas.

La integración educativa de las tecnologías recién está proyectándose, en este sentido, son los equipos docentes los que tenemos el desafío de asumir esta tarea transformadora en la educación matemática, ya que las nuevas generaciones lo reclaman. Debemos ser capaces de superar el fatalismo de la frase que se escucha a menudo, en el sentido que "tenemos universidades del siglo $\mathrm{XIX}$, con docentes del siglo $\mathrm{XX}$, para alumnos del siglo XXI".

\section{MATERIALES Y MÉTODOS}

Una de las principales realidades que vive el alumnado universitario, es su constante adaptabilidad al cambio tecnológico que se ofrecen en todas partes del mundo, computadoras de última generación, mini laptop, tabletas, Smartphone, son algunos de los dispositivos en el cual el estudiante accede al internet y que está sujeto a la "globalización tecnológica", pero en términos matemáticos simplemente se le conoce como "moda".

Partiendo de esta realidad, este estudio inició con un diagnóstico, cuyo propósito era conocer cuál era la red social que más utilizaban los estudiantes universitarios de la Facultad Regional Multidisciplinaria de Chontales, el uso que le daban cuando accedían a ella y su valoración al respecto de usar una red social para reforzar contenidos de las asignaturas que se impartían en el semestre de estudios, estas son algunas de sus opiniones:

\begin{tabular}{|c|c|c|c|c|c|c|c|}
\hline \multicolumn{2}{|c|}{ ¿Qué tipo de red social es la que más utilizas? } & \multirow{2}{*}{ Total } \\
\hline \multicolumn{2}{|c|}{} & Twitter & Facebook & MySpace & Otra & Ninguna & \\
\hline \multirow{2}{*}{ Sexo } & M & 8 & 55 & 0 & 6 & 4 & 73 \\
\cline { 2 - 7 } & F & 7 & 100 & 2 & 8 & 4 & 121 \\
\hline \multicolumn{2}{|c|}{ Total } & 15 & 155 & 2 & 14 & 8 & 194 \\
\hline
\end{tabular}

Tabla 1. Tipología en la utilización de redes sociales.

Claramente de observa que la red social que más utilizan los estudiantes es Facebook, representando un $80 \%$, con un amplio margen con relación a Twitter que aparece en segundo lugar (8\%).

Otra de las preguntas relevantes que se consideran en el diagnóstico consistió en saber la valoración a una idea innovadora para ayudar al reforzamiento de contenidos dados por los docentes, es gratificante saber que entre buena, muy buena y excelente idea suman un $80.92 \%$ de aceptación de ese proyecto, aunque existe un $19.07 \%$ que no le parece la idea, es comprensible pues no poseen cuenta en redes sociales o les parece que estas no son factibles para tal fin.

\section{¿Cómo categorizarías la idea de reforzar tus asignaturas usando Facebook?}

\begin{tabular}{|l|l|c|c|c|c|}
\hline \multicolumn{2}{|c|}{} & Frecuencia & $\begin{array}{c}\text { Porcen- } \\
\text { taje }\end{array}$ & $\begin{array}{c}\text { Porcentaje } \\
\text { válido }\end{array}$ & $\begin{array}{c}\text { Porcentaje } \\
\text { acumulado }\end{array}$ \\
\hline Validos & $\begin{array}{l}\text { Excelente } \\
\text { idea }\end{array}$ & 76 & 39.2 & 39.2 & 39.2 \\
\cline { 2 - 6 } & $\begin{array}{l}\text { Muy } \\
\text { buena } \\
\text { idea }\end{array}$ & 32 & 16.5 & 16.5 & 55.7 \\
\cline { 2 - 6 } & $\begin{array}{l}\text { Buena } \\
\text { idea }\end{array}$ & 49 & 25.3 & 25.3 & 80.9 \\
\cline { 2 - 6 } & Mala idea & 37 & 19.1 & 19.1 & 100.0 \\
\cline { 2 - 6 } & Total & 194 & 100.0 & 100.0 & \\
\hline
\end{tabular}

Tabla 2. Categorización del uso de Facebook como herramienta educativa. 
La información recepcionada a través del diagnóstico, ofreció la base para crear la página MATE AYUDA, en la red social Facebook, siguiendo los siguientes elementos:

- Facilidad en su uso.

- Actualización de contenido semanal.

- Apertura al diálogo entre los participantes.

- Envío y recepción de tareas.

- Peticiones de ayudas en diversas temáticas.

- Proponer temas de discusión.

Para conseguir los objetivos propuestos con la creación de la página, y como parte del camino que lleva la educación en nuestros tiempos, se hizo necesaria la introducción de esta herramienta tecnológica al alumnado, exponiéndoles que esta sería una forma de apoyo para que sus actividades académicas les resultarán mucho más fáciles de realizar y así, reforzar los aprendizajes matemáticos que se ofrecerán en las aulas escolares.

Para incentivar su participación, a menudo se colocaban problemas con un matiz diferente, pues se utilizan imágenes de series televisivas conocidas, ejercicios de razonamientos y propios de los temas de estudios de la asignatura, valorando permanentemente la calidad de sus intervenciones, su visión crítica y la capacidad para relacionar temas de la actualidad con las matemáticas. De este modo, se intenta implicar a los estudiantes en su propio aprendizaje, obligándoles a relacionar la materia de estudio con su realidad social.

Este trabajo, por sus características tiene un enfoque mixto, es cualitativo ya que estudia la realidad en su contexto natural, tal y como sucede, interpretando los fenómenos de acuerdo con los significados que tienen para las personas implicadas, además, produce datos descriptivos como las propias palabras de las personas, habladas o escritas, y la conducta observable. Es a la vez cuantitativo, porque permite examinar los datos de manera numérica, especialmente en el campo de la Estadística, haciendo demostraciones con los aspectos separados de su todo, a los que se asigna significado numérico y hace inferencias.

Por otro lado, el empleo de ambos procedimientos, cuantitativos y cualitativos, ayuda a corregir los sesgos propios de cada método, favoreciendo sus usos al "comunicarse con" y "servirse de" los sujetos del estudio (Cook y Retechardt, 2004). Además, el utilizar las fortalezas de ambos tipos de investigación combinándolas y tratando de minimizar sus debilidades potenciales, permiten que la recolección, análisis y vinculación de datos cualitativos y cuantitativos en un mismo estudio ofrezcan mejores resultados. ( Hernández, Fernández y Baptista, 2010)

Tiene carácter transversal, porque se hace un corte de tiempo único para describir o analizar el fenómeno, iniciando en agosto del 2013 y finalizando en noviembre del mismo año con la culminación de la asignatura.

El universo de este estudio estuvo conformado por 142 estudiantes, de las carreras de Ingeniería Agroindustrial, Bioanálisis Clínico y Administración de Empresas, todos de primer año, los cuales cursaban en ese momento, la asignatura Matemática General.

La muestra de 65 estudiantes fue seleccionada en forma no probabilística, realizándose por conveniencia. Se tomaron como criterios de selección las palabras escritas por los estudiantes en la página, sus opiniones, aportes, soluciones a problemas, sus envíos de tareas, y las visitas hechas a la página.

Sabiendo que la recogida de datos es un aspecto crucial dentro de la fase de investigación, por tanto, la selección de las técnicas de recopilación de datos queda a discreción del investigador, y refleja el nivel de involucramiento que el investigador va a asumir en el proceso de desarrollo de la investigación. Es por ello que Latorre (2003:54). recomienda: 
"El investigador ha de valorar que las técnicas que utiliza satisfagan las necesidades que el tema o problema de investigación plantea, y que sean eficaces para cubrir los objetivos formulados".

Bajo este antecedente, se utilizó una encuesta estructurada con 22 ítems para la recogida de información, los bloques de preguntas buscan conocer el grado de aceptación que tienen los estudiantes con relación a la página, sus valoraciones de la misma y autovaloraciones de su participación, y por último, la relevancia de usar Facebook en la enseñanza matemática.

- Para analizar los datos extraídos del instrumento investigativo, se siguieron los pasos siguientes:

- Primeramente se hizo una primera lectura para familiarizarse con los datos y tener un conocimiento aproximado sobre lo que se analizaría.

- Después, se hizo una base de datos en los que se fueron introduciendo las respuestas de los alumnos, para ello se utilizó el paquete estadístico SPSS versión 19.

- Una vez terminado este proceso, se analizaron los resultados a través de tablas de frecuencias y gráficos descriptivos, utilizando los procesadores de datos de Excel y SPSS.

- Se realizaron las inferencias respectivas, analizando los resultados obtenidos, acorde a lo descrito en las tablas y gráficos.

Por otro lado se tuvo en cuenta, los aportes dejados en la página por los estudiantes que hicieron uso de ella, al igual que sus reacciones ante frases, problemas y diversas situaciones dadas en la página.

\section{RESULTADOS}

Las opiniones dadas por parte de los estudiantes, permiten conocer el nivel de aceptación de la página como apoyo en la asignatura.

\section{a) Resultado del análisis cuantitativo:}

\section{¿EI nombre de la página MATE AYUDA va acorde} con lo que en ella se hace?

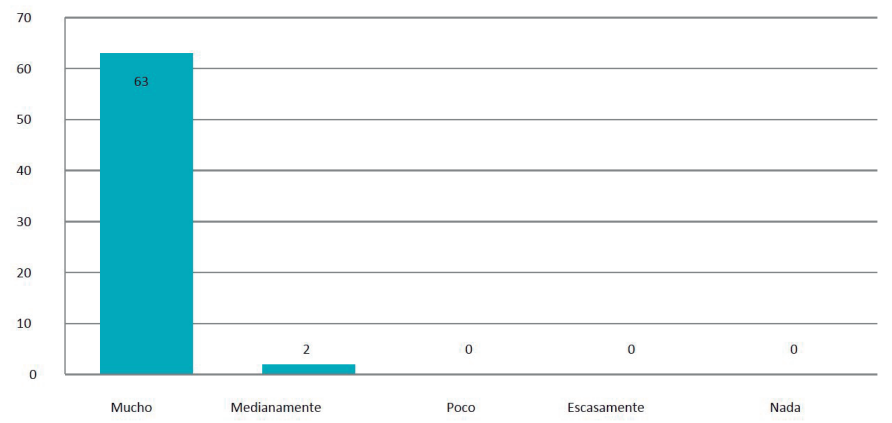

Figura 1. Concordancia de MATE AYUDA con su utilidad.

La figura 1 muestra el impacto positivo que ha causado el nombre de la página, por otro lado, se aprecia una clara relación entre el nombre de la página y lo que los estudiantes aseguran que se hace.

\section{¿Cuántas veces por semana revisas esta página?}

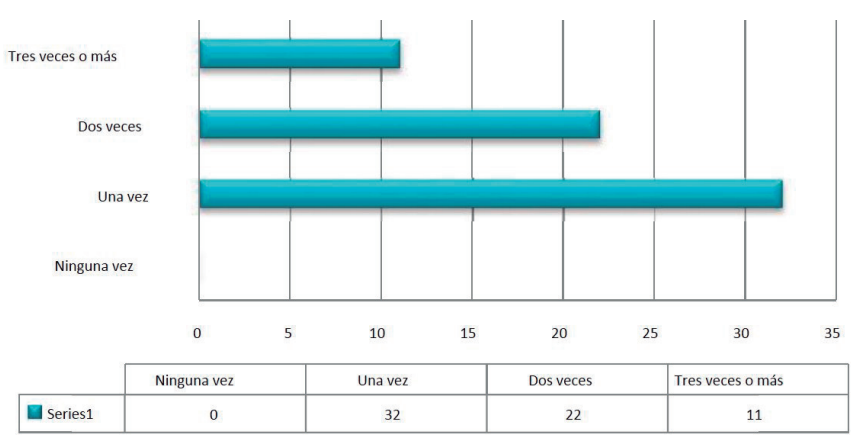

Figura 2. Visitas semanales realizadas. 
Es de notar que el $51 \%$ visita la página más de una vez, por lo que cada vez que se actualizaba el sitio, les aparecía la información en el muro y esto permitía que ellos accedieran a visualizar el contenido publicado.

\section{Cuando revisas la página ¿encuentras respuestas a tus inquietudes o dudas?}

Todas las veces

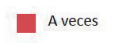

Muy pocas veces

No responden a mis dudas

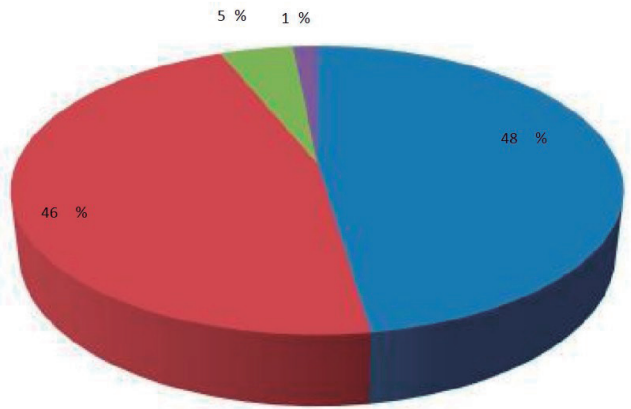

Figura 3.Valoración en relación a las respuestas dadas.

Es muy interesante constatar en la figura 3, que el $94 \%$ opinan que encuentran respuestas a sus dudas, atendiendo a los contenidos que fueron tratados en el aula de clase. En cambio, la figura 4 nos muestra que las publicaciones hechas en la página son suficientes para algunos, pero en algunos casos, los estudiantes piden que se publiquen la mayor cantidad de veces posibles.

¿Cúal es la utilidad más grande que le das a la página?
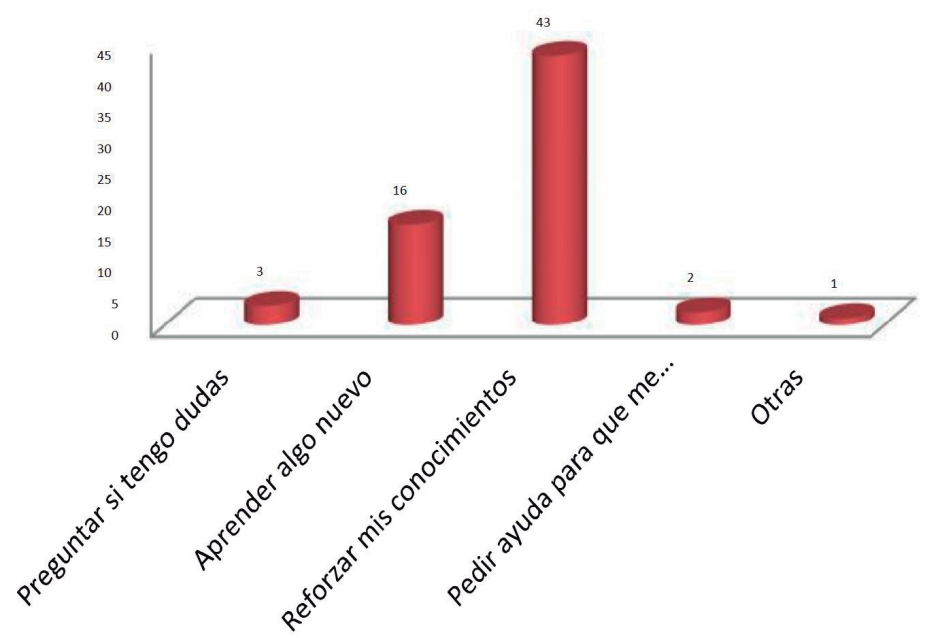

Figura 4. Utilidad que tuvo MATE AYUDA
A pesar que la página fue creada para todos los elementos que aparecen en la figura 5, buena parte de los estudiantes la utilizaron para reforzar conocimientos matemáticos y para aprender algo nuevo $(91 \%)$, claro está, que esta herramienta tecnológica permitió en gran medida que los mismos estudiantes se preocuparan por afianzar los conocimientos adquiridos en el salón de clase.

\section{¿La página colabora para que tus conocimientos adquiridos en matemática sean significativos?}

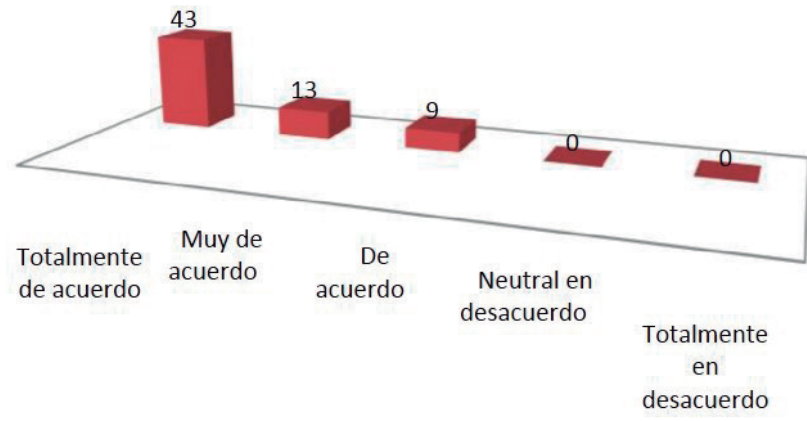

Figura 5. Significancia cognitiva de la página.

La figura anterior, muestra como Mate Ayuda colaboró en forma significativa en los aprendizajes alcanzados por los usuarios, manifestando de esta manera el apoyo que tuvieron con la apertura de la página.

\section{¿Cuál es tu valoración de la página MATE AYUDA?}

Excelente Muybuena Buena $\quad$ Regular $\quad$ Mala

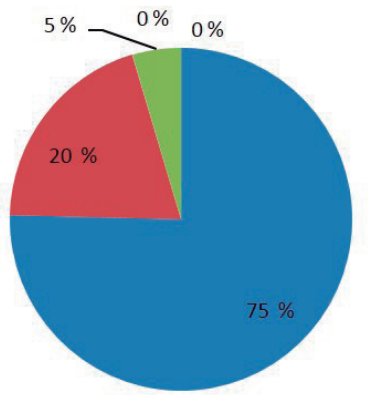

Figura 6. Valoración general de la Página. 
Es gratificante observar que los estudiantes valoraron excelentemente a la página con un $75 \%$, esto permite pensar que el objetivo novedoso de su creación causó muy buena impresión en los alumnos. El 91\% de los usuarios considera que MATE AYUDA se debe dejar en Facebook (figura 7), quizás por el amplio uso que se le da a esta red social entre el estudiantado universitario, o por la novedad de haber sido creada ahí y la facilidad de interactuar en ella.

De tener MATE AYUDA una segunda fase, ¿Dónde te gustaría que fuera creada?

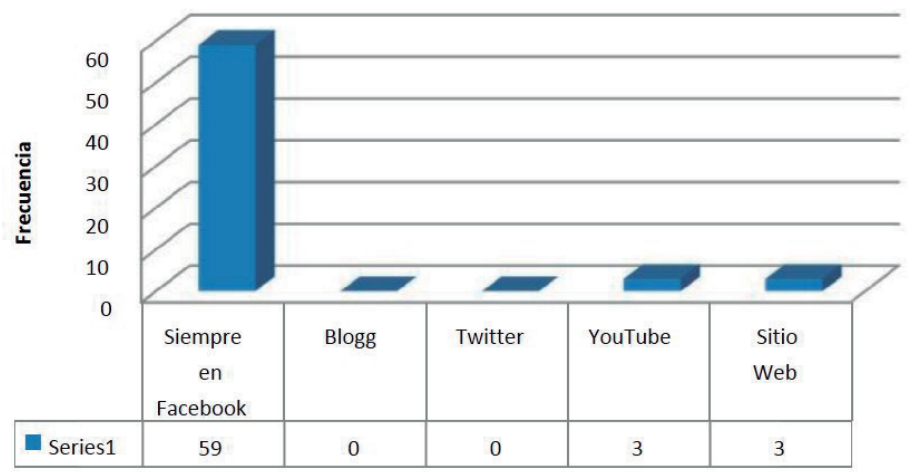

Figura 7. Propuesta de continuidad de la página.

Resulta interesante observar (figura 8 y 9) que los estudiantes aprueban la idea de usar redes sociales en el ámbito educativo, esto se debe quizás a varias razones: amplio uso de redes sociales, sencillez al utilizarlas y la interacción que se logra con ellas.

¿Cómo valoras la idea de usar Facebook con fines educativos?

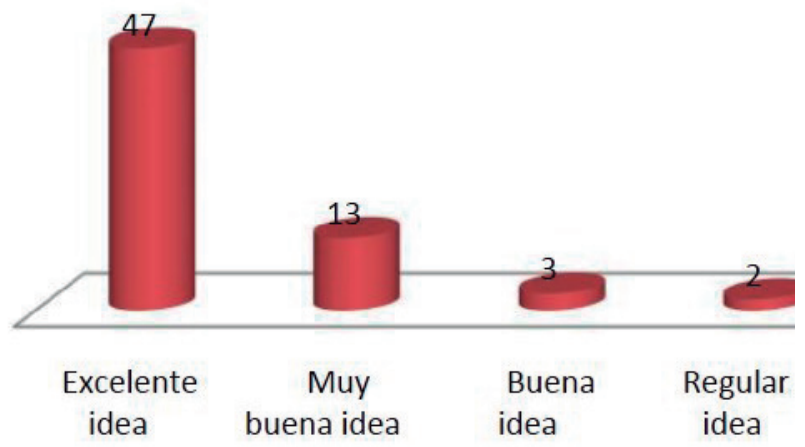

Figura 8. Valoración del uso de Facebook en educación.
¿Las redes sociales son una buena herramienta para mejorar la educación universitaria?

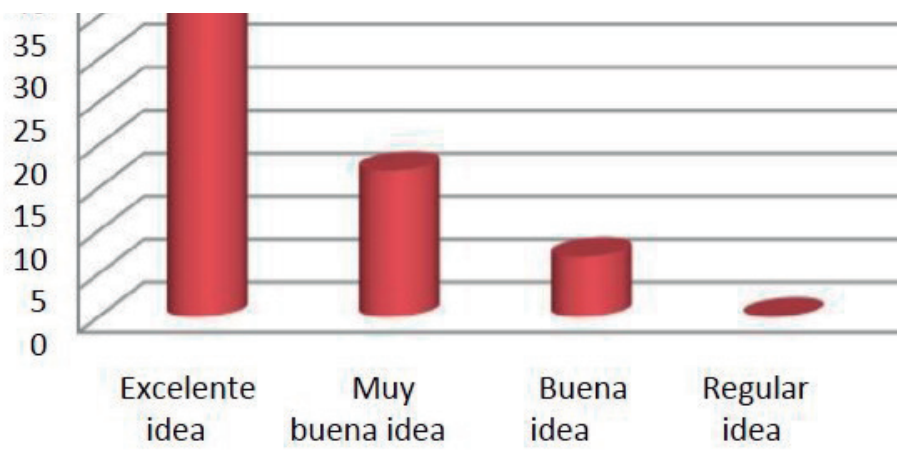

Figura 9. Valoración del uso de las redes sociales en la universidad.

\section{b) Resultado del análisis cualitativo:}

A continuación se presentan algunas interacciones, respuestas, solicitudes de ayuda y envíos de tareas hechas en la página (imagen 1, 2, y 3), con lo que se muestra la variedad de usos que se le dio a la misma. 


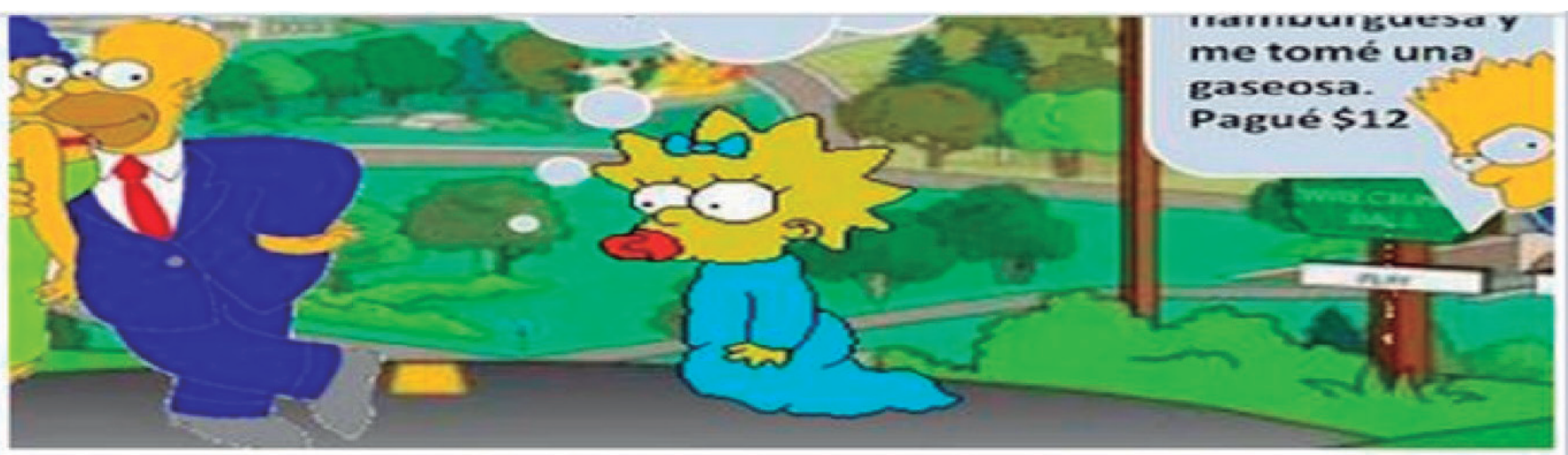

Me gusta - Comentar - Compartir

w3 A Isabel Ruiz, Humberto Ramon Rojas Murillo y 5 personas más les gusta esto.

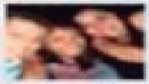

Zaydita Gomez 5 uazo cada gaseosa vale \$5 y cada hamburguesa $\$ 2$

9 de octubre a la(s) 20:10. Ya no me gusta * $\$ 2$

A I Felipe Torrez Reyes cada hamburguesa vale $\$ 5$ y cada gaseosa $\$ 2$.

9 de octubre a la(s) 20:41 * Va no me gusta * \$3 1

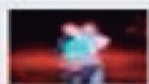

Belkyss Gisela Sevilla Amador Las hamburguesas 5 y cada gaseosa 2 no estudio Bioanalisis clinico pero tambien me queria divertir jijiji

9 de octubre a la(s) 20:54 * Va no me gusta * \& 1

Massiell Trafia Cada hamburguesa $\$ 5$ y cada gaseosa $\$ 2$

9 de octubre a la(s) 22:11 a través de celular " Ya no me gusta " 31

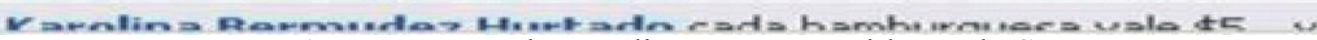

Imagen 1. Respuestas de estudiantes a un problema de SEL.

\section{Elizabeth Gonzalez}

\section{Elizabeth Gonzalez}

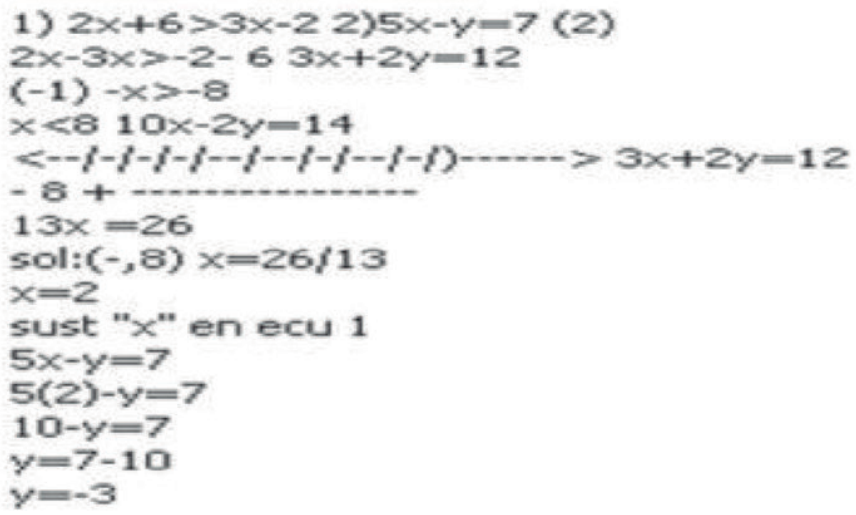

sol: $(2,-3)$

De: Elizabeth Precila Gonzales Arguello de Ing. Agroindustrial I ar̆io.

Imagen 2. Respuesta dada por una estudiante a un ejercicio de desigualdad lineal. 

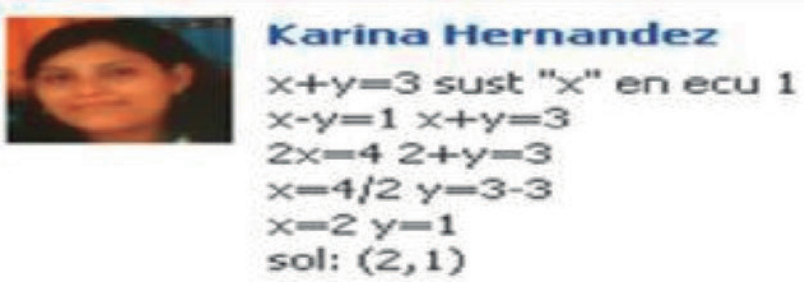

De: Martha Karina Leiva Hernández de Ing. Agroindustrial I ario.

Imagen 3. Secuencia de aportes brindada por una estudiante.

En muchas ocasiones, la página tenía un propósito motivacional para el estudiante, se les animaba a seguir adelante, ofreciendo consejos útiles para salir bien en la asignatura (imagen 4)

\section{tacebook}

Busca personas, lugares $y$ cosas

Est 6 s publicando, comentando e indicando que algo te gusta como MATE AVUDA - Cambi

\section{MATE AYUDA Biografia - Reciente -}

Estimados estudiantes de bioanálisis clínico, Administración de Empresas e Ingeniería Agroindustrial, les pedimos que este fir de semana aprovechen para estudiar y salir excelente en el examen. Recuerden que se debe esforzar para conseguir lo que se desea. Les dejamos una imagen alusiva a eso.

Saludos.

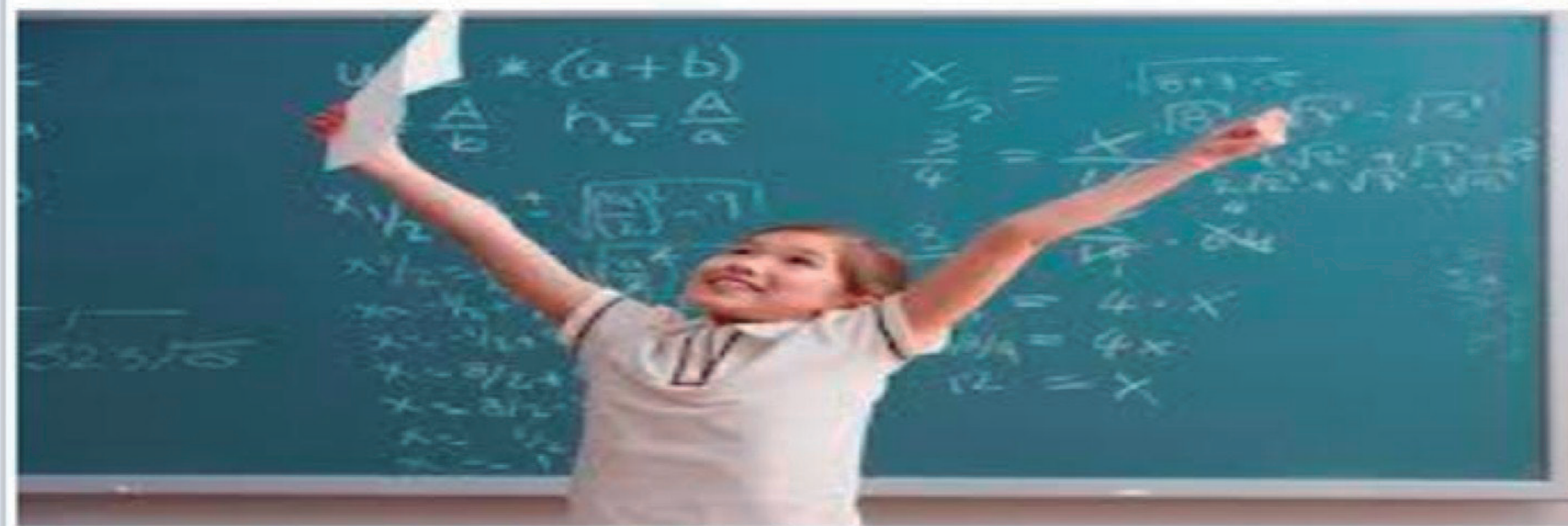

Me gusta - Comentar - Compartir

63 A Indiana Montoya Dompe, Winston Joseph Zamora Diaz, Velena Ar Guz $y$ a personas más les gusta esto.

Imagen 4. Aspecto motivacional inmerso en la página. 
Un aspecto que tomó mucho auge en la página es la solución a dudas o ejercicios que los estudiantes de las carreras enviaban, pero lo interesante de esto fue que no solamente ellos hacían preguntas, sino también estudiantes de secundaria y universitarios de este y otros países (imagen 5, 6 y 7).

\section{MATE AYUDA Mensajes -}

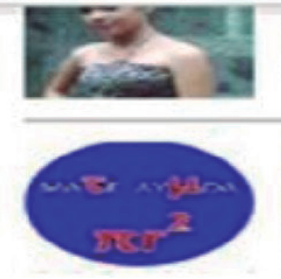

Me ayuda por favor??? La prof explico pero no le entendi.

Cual es el numero cuyo $3 / 4 \%$ son 21 ?

\section{MATE AYUDA}

El número se saca por regla de tres:

$\times-100 \%$

$21---3 / 4 \%$

Por lo tanto $x=[(21)(100)] / 3 / 4$

$x=2800$

Entonces el número cuyo $3 / 4 \%$ son 21 es 2800 .

Saludos Marifer

Marifer Jahaira Millon Hurtado

gracias saludos prof

Imagen 5. Solicitud y agradecimiento por una alumna de secundaria. 


\section{Publicaciones recientes de otras personas}

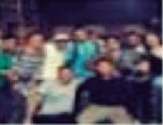

\section{Johangel Chirino}

hola ... necesito que me ayuden con un sistema de coordenadas polares ... pues resulta que intento encontrar los puntots de corte y no me da como en la grafica muestra... $3 \sqrt{3} \cos (\theta)=3 \operatorname{sen}(\theta)$

Me gusta * Comentar * 10 de octubre a la(s) 19:36

D MATE AYUDA Estimado Johangel, ya tu respuesta está publicada en la página, saludos.

11 de octubre a la(s) 17:17. Me gusta

Escribe un comentario...

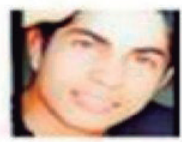

\section{Humberto Ramon Rojas Murillo}

wwwaaaoou que buena onda mate ayuda.....

Ya no me gusta . Comentar * 9 de octubre a la(s) 17:40

D. A MATE AYUDA le gusta esto.

Escribe un comentario...

\section{Magi Misukho}

oigan me podria ayudar a resolver esto es de operaciones

algebraicas... porfavor enserio necesito ayuda

En una tienda se compran tres diferentes articulos A, B, y C. A cuesta $3 x$ por unidad y se compran 5 unidades, B cuesta $4 x+2$ por unidad y se compran 3 unidades y $C$ cuesta $3 / 4 x$ por unidad y se compraron 7

Imagen 6. Solicitud de ayuda por parte de alumnos de otros países.

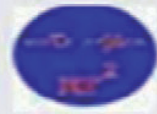

MATE AYUDA Que pasen felices fiestas navideñas y un buen año nuevo.

Hace aproximadamente una hora - Me gusta $\cdot \bowtie 1$

Carloos Dannieel Crusz Buenos días gracias por la ayuda me sirvió de mucho que se la pasen bien saludos desde Huauchinango Puebla

Hace aproximadamente una hora a través de celular · Me gusta

Imagen 7. Agradecimiento de un estudiante Mexicano. 


\section{CONCLUSIONES}

Es difícil suponer un encuentro no conflictivo entre la práctica educativa que ya no da respuesta a las necesidades estudiantiles y las posibilidades inmensas que brindan los saltos específicos provenientes de la cultura tecnológica. Actualmente se hace necesario inventar metodologías para todos los temas y niveles en las universidades, por tanto, al presentar en este artículo un Facebook lleno de opciones educativas, un Facebook como herramienta semiótica que puede facilitar aprendizajes en colaboración y un sitio que potencia la innovación en los procesos de enseñanza, el aprendizaje y la gestión del conocimiento a nivel individual y grupal, estamos promoviendo la cultura educativa del nuevo siglo.

Este trabajo al haberse planteado como objetivo, identificar el alcance que tiene Facebook al ser usado como un medio alterno e innovador para lograr afianzamientos de contenidos matemáticos en los estudiantes universitarios, y basado en toda la información recogida e interpretada, se presentan las siguientes conclusiones:

- La aceptación que tuvo el haber usado una red social en la educación de los estudiantes fue altamente positiva, reflejando que los jóvenes están abiertos al cambio de estilo en la enseñanza universitaria.

- El haber trabajado con la red social Facebook, permitió valorar el papel que juegan las TIC's y sus herramientas en la educación superior.

- El usar redes sociales, le permite al docente una cercanía con sus alumnos, ya que trasciende al ámbito del aula en todos los sentidos, obteniendo así, más retroalimentación en forma autónoma.

- Facebook promueve la conversación de manera espontánea, generando avisos automáticos de publicaciones en el muro, permitiendo ser revisados incluso, desde los teléfonos celulares.

- Al utilizar Facebook en el ámbito educativo, se rompe el esquema relacionado a su uso, ya no solamente como un instrumento de ocio o de comunicación social, sino también como una herramienta educativa y de uso profesional.

- Al interactuar educacionalmente usando Facebook, el principal papel del profesor es como guía y moderador del grupo, permitiendo de esta manera orientar en forma dinámica a cada participante de la página.

- Utilizar Facebook en el sistema de enseñanza y aprendizaje universitario no debe ser visto como un fin, sino como un medio. Se debe enseñar con tecnologías, no enseñar tecnologías solamente.

- El trabajar con redes sociales implica una constante planificación, no sólo de los materiales que serán publicados, sino de la manera en que se pretende obtener el objetivo educativo, esto implica actividades concretas y la moderación correcta de dichas actividades.

- Como espacio de participación colaborativa estudiantil, este tipo de recursos es muy valioso, por ser un espacio de aprendizaje y una herramienta complementaria, valdría la pena contrastarla con una plataforma educativa en Moodle.

- Se debe aprovechar el impacto que tienen las TIC's en educación, debido a que brinda las oportunidades de adecuar nuestra forma de enseñanza, y por ende estar al día con los usos que se le puede dar a las nuevas tecnologías.

- A los estudiantes les resulta agradable la idea de tener espacios virtuales donde practicar lo aprendido, revisar lecciones y realizar deberes, no sólo en matemática, sino también en otras asignaturas.

- La oportunidad de utilizar videos como un medio para enseñar contenidos matemáticos, permite a los estudiantes estudiar en casa lo que no se logró comprender bien en el salón de clases.

- La página MATEAYUDA, ha probado ser efectiva para motivar el interés por la asignatura, para auto prepararse ante evaluaciones y para reforzar temáticas propias del programa de estudio, siendo 
esta última la principal razón de visita por los estudiantes.

- Al ser la mayoría de estudiantes usuarios activos de Facebook, proponen que la página siga existiendo en esa misma plataforma virtual, ya que consideran que se les hace fácil obtener las actualizaciones de la página a través del muro de la misma.

- El haber utilizado los intereses y motivaciones de los mismos estudiantes y la gama de contextos para aprender matemática, facilitó las visitas constantes de los estudiantes en la página.

- La página fue útil no solamente para los involucrados en ésta investigación, sino también para docentes, estudiantes de modalidades de secundaria y universitaria de nuestro país, al igual que de otros países.

\section{BIBLIOGRAFÍA}

Cook, T. Retechardt, C. (2004). "Métodos Cuantitativos y Cualitativos en investigación Educativa". Madrid: Morata.

Barajas, F. Álvarez, C. (2013). "Uso de Facebook como herramienta en la enseñanza del área de naturales en el grado undécimo de educación media vocacional”. [Versión electrónica]. Pixel-Bit. Revista de medios y educación.(42),143-156.

Font, V. Godino, J. (2011). Inicio a la investigación en la enseñanza de las Matemáticas en secundaria y bachillerato. En Goñi, J. (coord.) "Matemáticas: Investigación, innovación y buenas prácticas". Barcelona: Graó; 9-52.

Freire, P. (2005). "Pedagogía del oprimido". México, D.F: Siglo XXI editores; 75-103.

García, I. Peña, I. Johnson, L. Smith, R. Levine, A. y Haywood, K. (2010). "Informe Horizon: Edición Iberoamericana 2010". Austin: The New Media Consortium; 3-15.

Gómez, M. López, N. (2010) . “Uso de Facebook para actividades académicas colaborativas en educación media y universitaria". Recuperado el 15 de octubre de 2013 de: http://goo.gl/epL2j Hernández, R. Fernández, C. Baptista, P. (2010). "Metodología de la investigación". México: McGraw-Hill.

Latorre, A. (2003)."La investigación-acción”. Barcelona: Graó; 52-57

López, F. (2007). "Metodología Participativa en la Enseñanza Universitaria".

Madrid: Narcea; 15-19 Orihuela, J. (2008). "Internet: la hora de las redes sociales". Nueva Revista (119); 57-62. Recuperado el 17 de agosto de 2013 de: http://goo.gl/XDRV1n

Planas, N. (2011). Buenas prácticas en la enseñanza de las matemáticas en secundaria y bachillerato. En Goñi, J. (coord.) "Matemáticas: Investigación, innovación y buenas prácticas". Barcelona: Graó; 57-157.

Santamaría, F. (2009). Redes sociales y comunidades educativas. Posibilidades pedagógicas. "Telos: cuadernos de comunicación e innovación”. (76); 99-109. Recuperado el 17 de agosto de 2013, de: http://goo.gl/TV798

Solana, F. (2008). “Educar ¿Para qué?”. México, D.F: Grupo Noriega Editores; 79-83

TED. (2011). "Salman Khan. Usemos el video para reinventar la educación". Recuperado el 11 de noviembre de 2013 de: http://goo.gl/HmCGN Tünnermann, C. (1993). "Perspectivas del desarrollo de la educación superior en Nicaragua". Managua: Nueva Nicaragua; 230-234.

Zamora, W. (2013). "Hacia la innovación en la educación superior de Nicaragua: espacios para la reflexión y el desarrollo". [Versión electrónica]. Revista UNAN-FAREM Estelí. 2 (6); 1-9. 